\title{
Fabrication of aligned Nanowire Device using Contact Printing Method
}

\author{
Youngsik Kim ${ }^{1}$, Yongho $\mathrm{Choi}^{2}$ and Youngsam $\mathrm{Lim}^{3 *}$ \\ ${ }^{1}$ Department of Elementary Education, Korea National University of Education, \\ Cheongju-si, South Korea \\ ${ }^{2}$ Department of Aero-Materials Engineering, Jungwon University, Goesan-eup, \\ South Korea \\ ${ }^{3}$ Department of Sport Industry Major, Jungwon University, Goesan-eup, \\ South Korea \\ ys-kim@knue.ac.kr,yhchoi@jwu.ac.kr, "lysgolf@jwu.ac.kr
}

\begin{abstract}
It is quite challenging to fabricate individual nanomaterial devices due to its small dimensions. So far, most of researches have focused on the two methods to fabricate the individual nanomaterial devices. One of the methods is growing individual nanomaterials on desired locations. The other one is growing large numbers of nanomaterials and then etching most of undesired nanomaterials. In this study, we used a simple method to fabricate individual nanowire devices based on a contact printing method. The method enables direct transferring and positioning nanowires to a receiver substrate with a good alignment. This study could be useful to fabricate individual nanomaterials devices and also could be extended to the fabrication of nanomaterials composites such as golf shaft due to good alignment of nanomaterials resulting in better mechanical properties.
\end{abstract}

Keywords: Contact printing, GaN nanowire, Vapour-Liquid-Solid, Device Fabrication, Nanowire Alignment

\section{Introduction}

1-Dimensional nanomaterials such as nanowires and nanotubes have been intensively studied during past decades due to their outstanding properties due to their low dimensionality [1-7]. Based on their properties, the nanomaterials have been considered and explored as the potential materials of future applications in integrated electronic, optoelectronic, bio-sensing, and photonic devices [2, 3, 5-18].

The one of the technical problems to implement nanomaterials in devices is the handling issue. To integrate nanomaterials with other materials, it is frequently demanded to control their location, alignment and density. However, as-grown nanomaterials by common growth methods are generally high dense bundles and randomly oriented. Many efforts have been made with the issues and which resulted in several techniques to fabricate individual nanomaterial devices with proper orientations $[4,8,19-23]$.

For example, nanomaterials were firstly collected from grown substrate, and then transferred and aligned on a target substrate using electric, magnetic, microfluidic, or molecular forces.

For the other method, catalysts were patterned before growth to innately localize growing nanomaterials. Recently, direct contact printing method was reported [24-

Received (October 7, 2017), Review Result (December 1, 2017), Accepted (December 5, 2017)

* Corresponding Author 
26]. The method is relatively more convenient, efficient, and fast process compared to other techniques. Also, some processes which are required in the other methods such as collecting and sonicating nanowires were not necessary in the method.

Moreover, it was resulted in highly ordered and well aligned nanowires. So far, several electronic devices have been designed, fabricated, and characterized using the method [24-26]. In this study, we demonstrate that the contact printing method can be used for fabrication of aligned nanowire devices. This study could be also useful to fabricate nanomaterials composites such as golf shaft for better mechanical properties due to good alignment.

\section{Experimental Methods}

GaN nanowires used in this study were prepared by Vapour-Liquid-Solid (VLS) growth method [27]. In brief, firstly, a 500-nm-thick $\mathrm{SiO}_{2}$ layer was thermally grown on silicon substrates. As a catalyst, $\sim 1 \mathrm{~nm}$ Au layer was deposited on $\mathrm{SiO}_{2}$ surface by e-beam evaporation. The liquid gallium metal was put into a quartz boat as a Ga source and placed into a 1-inch atmospheric quartz tube.

The $\mathrm{Au}$ thin film catalyst deposited substrate was placed to $\sim 3 \mathrm{~cm}$ away (downstream) from the gallium metal source. A $500 \mathrm{sccm}$ Ar was introduced into the quartz tube and the substrate was then heated to $850^{\circ} \mathrm{C}$ and annealed at the temperature for 15 min with the Ar flow. During this annealing period, Au thin film was aggregated and forms discrete Au nanoparticles on the $\mathrm{SiO}_{2}$ surface. After the annealing period, the growth of $\mathrm{GaN}$ nanowires was performed at $850^{\circ} \mathrm{C}$ for 2 hours with a flow of $\mathrm{NH}_{3}$ and $\mathrm{H}_{2}$ with a flow rate of $15 \mathrm{sccm}$ and $300 \mathrm{sccm}$, respectively.

Finally, the substrate was taken out from the furnace after it was cooled down to room temperature.

The as-grown GaN nanowires were characterized by a JEOL JSM-6335F fieldemission gun scanning electron microscope (SEM), a JEOL 2010F high resolution transmission electron microscope (HRTEM) operating at $200 \mathrm{kV}$. For the HRTEM characterization, the as-grown nanowires were dispersed in 2-propanol followed by a sonication, and then a few drops of the nanowire solution were made onto a commercial TEM grid.

The as-grown GaN nanowires were directly transferred and aligned on a target substrate by a simple contact printing method. Figure 1 is a schematic diagram of the method. Firstly, the sample containing nanowires, donor substrate, was inverted and placed on a target sample, receiver substrate. Secondary, to apply constant pressures, weights were attached on a back side of the donor substrate.

Finally, the donor substrate moved in one direction at a constant speed on the receiver substrate. 


\section{Donor substrate}
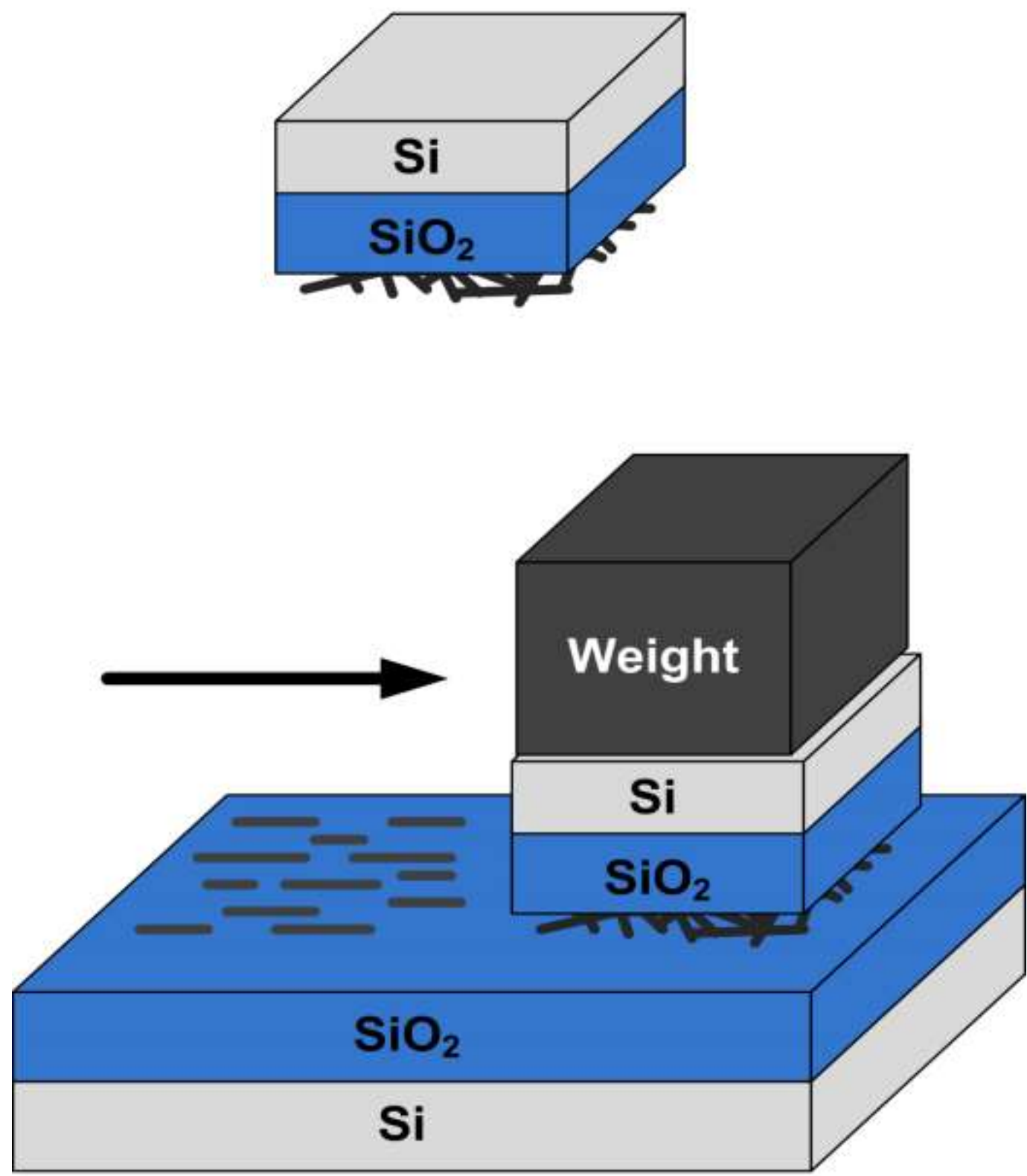

\section{Receiver substrate}

Figure 1. A Schematic Diagram of a Contact Printing Method

\section{Results and discussion}

Figure 2 (a) and (b) are SEM and TEM image of as-grown GaN nanowires, respectively. High density of nanowires was grown and they were randomly oriented as shown in the SEM image. Lengths of nanowires are in the range of $1 \mathrm{um}$ to $20 \mathrm{um}$ with diameters of $10 \mathrm{~nm}$ to $60 \mathrm{~nm}$ characterized by SEM and TEM images. 

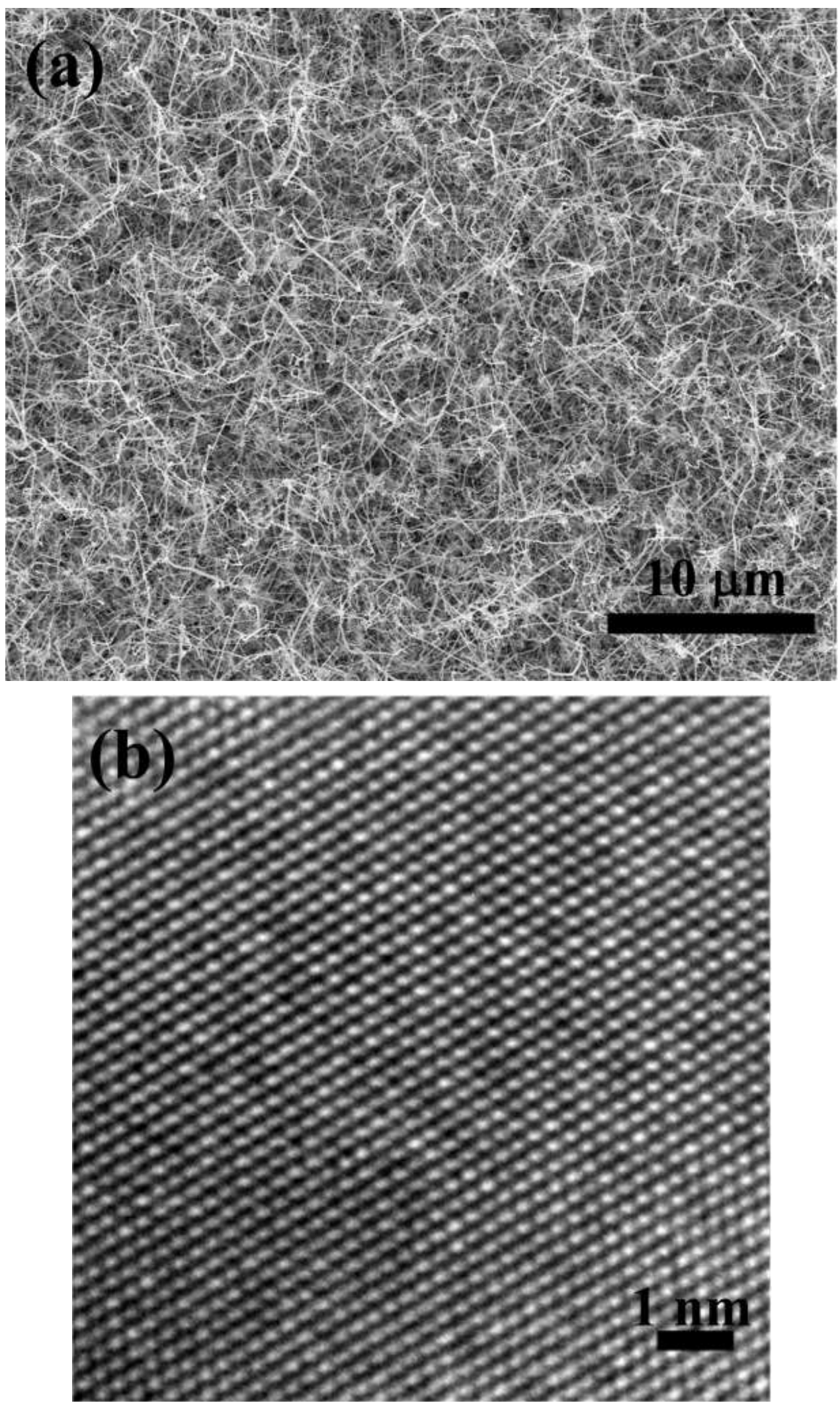

\section{Figure 2. (a) SEM and (b) TEM Image of as-grown GaN nanowires}

The density of transferred nanowires was increased by increasing the pressure applied on a donor substrate. The lengths of nanowires were much shortened, also. It is most likely due to larger friction forces between nanowires and/or between 
nanowires and substrates. The heavier weight increased the friction forces during travelling of donor substrate which resulted in transferring more nanowires and breaking off the transferring nanowires as well. Some lubricants can be used between a donor and a receiver substrate for the better alignment of nanowires which could be due to reduced friction forces [25]. Next, we applied a finger force on a donor substrate.

The donor substrate was pressed down by a finger and we assumed that much larger pressure was applied. The quite large numbers of nanowires were transferred at certain places and they were congregated. A higher magnification SEM image, which is not included, showed that nanowires were highly dense and randomly oriented, and that was very similar with the as-grown nanowires. As a result, density and alignment of transferring nanowires are sensitively affected by the pressure on the donor substrate during contact printing, and it should be carefully adjusted. We transferred nanowires with increased sliding speed of donor substrate. (The weight on the donor substrate was $7 \mathrm{~g}$.)

The speed for the previous experiments was $\sim 20 \mathrm{um} / \mathrm{sec}$, and it was increased to $\sim 100 \mathrm{um} / \mathrm{sec}$. The relatively faster movement of donor substrate resulted in worse alignment and higher density of nanowires. It is most likely due to an insufficient time for alignment.

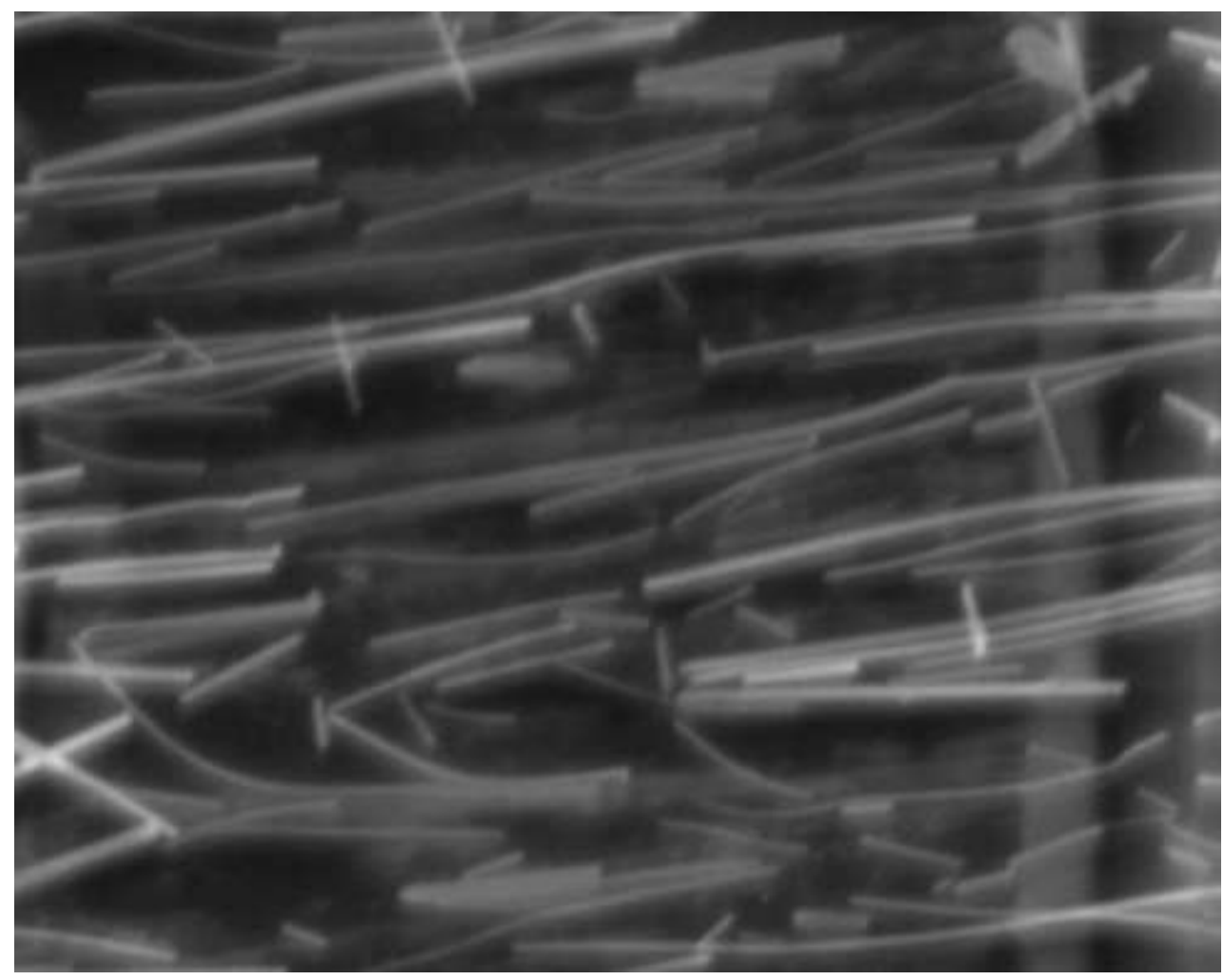

Figure 3. SEM Image of Transferred Nanowires using Contact Printing

During transferring of nanowires, the one end of nanowires was attached to the surface of receiver substrate and the other end was pulled by donor substrate moving. However, the faster movement may cause divergent movement the one end of nanowires not following the direction of donor substrate. In this case, the higher density of transferred nanowires was most likely due to an increasing the friction forces caused by the faster movement as similar as the higher-pressure case. 
Therefore, slower speed is desirable for better alignment and less dense of nanowires on the target substrate.

It should be noted that a density of transferred nanowires is also affected by density of nanowires on a donor substrate, as well as a pressure and a movement speed of a donor substrate.

The density of transferred nanowires was significantly reduced if a lower density donor substrate used. Therefore, the pressure and the movement speed for donor substrate should be adjusted based on conditions of donor substrate such as a nanowire density.

\section{Conclusion}

We have demonstrated that the density and alignment of transferred nanowires for contact printing method are sensitively affected by the pressure and the movement speed of donor substrate. The densities of nanowires were successfully adjusted with contact printing method. The results indicate that aligned nanowire devices can be fabricated in various structures using the simple contact printing method and it is useful for future applications.

\section{References}

[1] B. Q. Zhu, G. P. Wei, X. C. Li, Z. Ma and Y. Wei, "Preparation and growth mechanism of carbon nanotubes via catalytic pyrolysis of phenol resin", Materials Research Innovations, vol. 18, (2014), pp. 267-272.

[2] Z. Chen, J. Appenzeller, Y.-M. Lin, J. Sippel-Oakley, A. G. Rinzler, J. Tang, S. J. Wind, P. M. Solomon, and P. Avouris, "An Integrated Logic Circuit Assembled on a Single Carbon Nanotube", Science, vol. 311, (2006), pp. 1735.

[3] R. S. Friedman, M. C. McAlpine, D. S. Ricketts, D. Ham and C. M. Lieber, "Nanotechnology Highspeed integrated nanowire circuits", Nature, vol. 434, (2005), pp. 1085-1085.

[4] Y. Huang, X. Duan, Y. Cui, L. J. Lauhon, K.-H. Kim and C. M. Lieber, "Logic Gates and Computation from Assembled Nanowire Building Blocks", Science, vol. 294, (2001), pp. 1313-1317.

[5] A. Javey, J. Guo, Q. Wang, M. Lundstrom and H. Dai, "Ballistic carbon nanotube field-effect transistors", Nature, vol. 424, (2003), pp. 654-657.

[6] A. Javey, H. Kim, M. Brink, Q. Wang, A. Ural, J. Guo, P. McIntyre, P. McEuen, M. Lundstrom and H. Dai, "High-[kappa] dielectrics for advanced carbon-nanotube transistors and logic gates", Nat Mater, , vol. 1, (2002), pp. 241-246.

[7] X. Wang, J. Song, J. Liu and Z. L. Wang, "Direct-Current Nanogenerator Driven by Ultrasonic Waves", Science, vol. 316, (2007), pp. 102-105.

[8] J.-H. Ahn, H.-S. Kim, K. J. Lee, S. Jeon, S. J. Kang, Y. Sun, R. G. Nuzzo and J. A. Rogers, "Heterogeneous Three-Dimensional Electronics by Use of Printed Semiconductor Nanomaterials", Science, vol. 314, (2006), pp. 1754-1757.

[9] T. Bryllert, L. E. Wernersson, L. E. Froberg and L. Samuelson, "Vertical high-mobility wrap-gated InAs nanowire transistor", Electron Device Letters, IEEE, vol. 27, (2006), pp. 323-325.

[10] Y. Cui, Q. Wei, H. Park and C. M. Lieber, "Nanowire Nanosensors for Highly Sensitive and Selective Detection of Biological and Chemical Species", Science, vol. 293, (2001), pp. 1289-1292.

[11] S. Ju, A. Facchetti, Y. Xuan, J. Liu, F. Ishikawa, P. Ye, C. Zhou, T. J. Marks and D. B. Janes, "Fabrication of fully transparent nanowire transistors for transparent and flexible electronics", Nat Nano, vol. 2, (2007), pp. 378-384.

[12] N. S. Lewis, "Toward Cost-Effective Solar Energy Use", Science, vol. 315, (2007), pp. 798-801.

[13] F. Patolsky, B. P. Timko, G. Yu, Y. Fang, A. B. Greytak, G. Zheng and C. M. Lieber, "Detection, Stimulation, and Inhibition of Neuronal Signals with High-Density Nanowire Transistor Arrays", Science, vol. 313, (2006), pp. 1100-1104.

[14] E. Stern, J. F. Klemic, D. A. Routenberg, P. N. Wyrembak, D. B. Turner-Evans, A. D. Hamilton, D. A. LaVan, T. M. Fahmy and M. A. Reed, "Label-free immunodetection with CMOS-compatible semiconducting nanowires", Nature, vol. 445, (2007), pp. 519-522.

[15] D. Wang, B. A. Sheriff and J. R. Heath, "Complementary Symmetry Silicon Nanowire Logic: PowerEfficient Inverters with Gain", Small, vol. 2, (2006), pp. 1153-1158.

[16] J. Wang, M. S. Gudiksen, X. Duan, Y. Cui and C. M. Lieber, "Highly Polarized Photoluminescence and Photodetection from Single Indium Phosphide Nanowires", Science, vol. 293, (2001), pp. 1455-1457.

[17] Z. L. Wang and J. Song, "Piezoelectric Nanogenerators Based on Zinc Oxide Nanowire Arrays", Science, vol. 312, (2006), pp. 242-246. 
[18] D. Zhang, Z. Liu, C. Li, T. Tang, X. Liu, S. Han, B. Lei and C. Zhou, "Detection of NO2 down to ppb Levels Using Individual and Multiple In2O3 Nanowire Devices", Nano Letters, vol. 4, (2004), pp. 19191924.

[19] S. Jin, D. Whang, M. C. McAlpine, R. S. Friedman, Y. Wu and C. M. Lieber, "Scalable Interconnection and Integration of Nanowire Devices without Registration", Nano Letters, vol. 4, (2004), pp. 915-919.

[20] K. Keren, R. S. Berman, E. Buchstab, U. Sivan and E. Braun, "DNA-Templated Carbon Nanotube FieldEffect Transistor", Science, vol. 302, (2003), pp. 1380-1382.

[21] P. J. Pauzauskie, A. Radenovic, E. Trepagnier, H. Shroff, P. Yang and J. Liphardt, "Optical trapping and integration of semiconductor nanowire assemblies in water", Nat Mater, vol. 5, (2006), pp. 97-101.

[22] S. G. Rao, L. Huang, W. Setyawan and S. Hong, "Nanotube electronics: Large-scale assembly of carbon nanotubes", Nature, vol. 425, (2003), pp. 36-37.

[23] G. Yu, A. Cao and C. M. Lieber, "Large-area blown bubble films of aligned nanowires and carbon nanotubes", Nat Nano, (2007), vol. 2, pp. 372-377.

[24] Z. Fan, J. C. Ho, Z. A. Jacobson, H. Razavi and A. Javey, "Large-scale, heterogeneous integration of nanowire arrays for image sensor circuitry", Proceedings of the National Academy of Sciences, vol. 105, (2008), pp. 11066-11070.

[25] Z. Fan, J. C. Ho, Z. A. Jacobson, R. Yerushalmi, R. L. Alley, H. Razavi and A. Javey, "Wafer-Scale Assembly of Highly Ordered Semiconductor Nanowire Arrays by Contact Printing", Nano Letters, vol. 8, (2007), pp. 20-25.

[26] A. Javey, Nam, R. S. Friedman, H. Yan and C. M. Lieber, "Layer-by-Layer Assembly of Nanowires for Three-Dimensional, Multifunctional Electronics", Nano Letters, vol. 7, (2007), pp. 773-777.

[27] J. L. Johnson, Y. Choi and A. Ural, Journal of Vacuum Science \& Technology B, vol. 26, (2008) 
International Journal of Control and Automation

Vol. 10, No. 12 (2017) 